\title{
An integral field spectrograph for SNAP
}

\section{Anne Ealet, Eric Prieto, Alain Bonissent, Roger Malina, Gérard Smadja, et al.}

Anne Ealet, Eric Prieto, Alain Bonissent, Roger Malina, Gérard Smadja, A. Tilquin, Gary Bernstein, Stephane Basa, D. Fouchez, Olivier Le Fevre, Alain Mazure, Greg Aldering, R. Amanullah, Pierre Astier, E. Barrelet, Christopher J. Bebek, Lars Bergstrom, Manfred Bester, Roger Blandford, Ralph C. Bohlin, Charles R. Bower, Mark L. Brown, Myron Campbell, William C. Carithers, Eugene D. Commins, W. Craig, C. Day, F. DeJongh, Susana E. Deustua, H. T. Diehl, S. Dodelson, Richard S. Ellis, M. Emmet, Josh Frieman, Andrew Fruchter, D. Gerdes, L. Gladney, Gerson Goldhaber, Ariel Goobar, Donald E. Groom, Henry D. Heetderks, M. Hoff, Stephen E. Holland, M. Huffer, L. Hui, Dragan Huterer, B. Jain, Patrick N. Jelinsky, Armin Karcher, Steven M. Kent, Steven M. Kahn, Alex G. Kim, William F. Kolbe, B. Krieger, G. Kushner, N. Kuznetsova, Robin E. Lafever, J. I. Lamoureux, Michael L. Lampton, Michael E. Levi, P. Limon, Huan Lin, Eric V. Linder, Stewart C. Loken, W. Lorenzon, J. Marriner, P. Marshall, R. Massey, Timothy A. McKay, Shawn P. McKee, Ramon Miquel, Nicholas Morgan, E. Mörtsell, Nick Mostek, Stuart Mufson, J. A. Musser, Peter E. Nugent, Hakeem M. Oluseyi, Reynald Pain, Nick P. Palaio, David H. Pankow, John Peoples, Saul Perlmutter, David Rabinowitz, Alexandre Refregier, Jason Rhodes, Natalie A. Roe, D. Rusin, V. Scarpine, Michael S. Schubnell, Michael J. Sholl, Roger M. Smith, George F. Smoot, Jeffrey A. Snyder, Anthony Spadafora, A. Stebbins, Christopher Stoughton, Andrew Szymkowiak, Gregory Tarlé, Keith Taylor, Andrew D. Tomasch, Douglas Tucker, Henrik von der Lippe, D. Vincent, Jean-Pierre Walder, Guobin Wang, W. Wester, "An integral field spectrograph for SNAP," Proc. SPIE 5487, Optical, Infrared, and Millimeter Space Telescopes, (12 October 2004); doi: 10.1117/12.550600 


\title{
An Integral Field Spectrograph for SNAP
}

A. Ealet ${ }^{\mathrm{g}, \mathrm{m}}$; E. Prieto ${ }^{\mathrm{m}}$; A. Bonissent ${ }^{\mathrm{g}} ;$ R. Malina ${ }^{\mathrm{m}} ;$ G. Smadja $^{\mathrm{p}}$, A.Tilquin ${ }^{\mathrm{g}}$, G. Bernstein ${ }^{\mathrm{e}}$, S. Basa ${ }^{\mathrm{m}}$, D.Fouchez ${ }^{\mathrm{g}}$, O. LeFevre ${ }^{\mathrm{m}}$, A. Mazure ${ }^{\mathrm{m}}$,

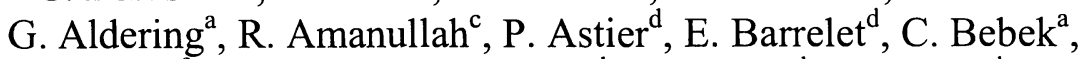
L.Bergstr ${ }^{\mathrm{c}}$, M. Bester ${ }^{\mathrm{f}}$, R.Blandford ${ }^{\mathrm{q}}$, R.Bohlin ${ }^{\mathrm{k}}$, C. Bower ${ }^{\mathrm{h}}$, M.Brown $^{\mathrm{b}}$, M.Campbell ${ }^{\mathrm{b}}$, W. Carithers ${ }^{\mathrm{a}}$, E. Commins ${ }^{\mathrm{f}}$, W.Craig ${ }^{\mathrm{q}}$, C. Day ${ }^{\mathrm{a}}$, F.DeJongh ${ }^{r}$, S. Deustua ${ }^{\mathrm{i}}$, H.T.Diehl ${ }^{\mathrm{r}}$, S.Dodelson ${ }^{r}$, R. Ellis ${ }^{j}$, M. Emmet ${ }^{\mathrm{s}}$, J.Frieman ${ }^{\mathrm{r}}$, A. Fruchter ${ }^{\mathrm{k}}$, D.Gerdes ${ }^{\mathrm{b}}$, L.Gladney ${ }^{\mathrm{e}}$,

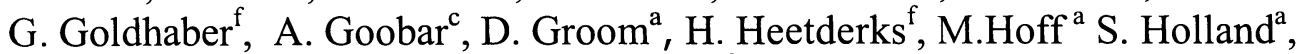
M.Huffer ${ }^{\mathrm{q}}$, L.Huil ${ }^{\mathrm{r}}$, D. Huterer ${ }^{1}$, B.Jain ${ }^{\mathrm{e}}$, P.Jelinsky ${ }^{\mathrm{f}}$, A. Karcher ${ }^{\mathrm{a}}$, S.Kent ${ }^{\mathrm{r}}, \mathrm{S} . \mathrm{Kahn}^{\mathrm{q}}$, A. Kim ${ }^{\mathrm{a}}$, W. Kolbe ${ }^{a}$, B. Krieger ${ }^{a}$, G.Kushner ${ }^{a}$, N.Kuznetsova ${ }^{a}$, R. Lafever ${ }^{a}$, J. Lamoureux ${ }^{a}$, M. Lampton,

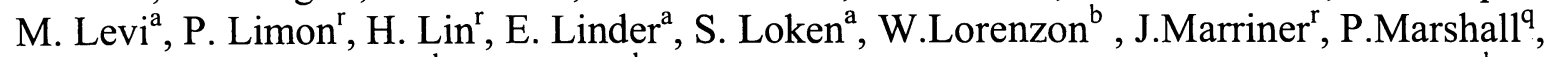

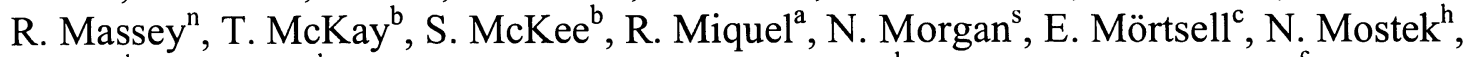



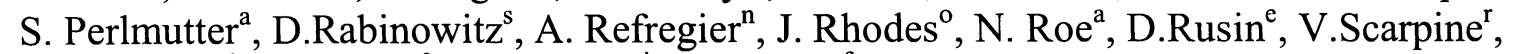
M. Schubnell ${ }^{\mathrm{r}}$, M. Sholl ${ }^{\mathrm{f}}$, R.M.Smith ${ }^{\mathrm{j}}$, G. Smoot ${ }^{\mathrm{f}}$, J.Snyder ${ }^{\mathrm{s}}$, A. Spadafora ${ }^{\mathrm{a}}$, A.Stebbins ${ }^{\mathrm{r}}$, C.Stoughton ${ }^{r}$, A. $\sim$ Szymkowiak $^{\mathrm{s}}$, G. Tarle ${ }^{\mathrm{b}}$, K.Taylor ${ }^{\mathrm{j}}$, A. Tomasch ${ }^{\mathrm{b}}$, D.Tucker ${ }^{\mathrm{r}}$, H.von der Lippe ${ }^{\mathrm{a}}$, D. Vincent ${ }^{\mathrm{d}}, \mathrm{J}$-P. Walder ${ }^{\mathrm{a}}$, G. Wang ${ }^{\mathrm{a}}$, W.Wester ${ }^{\mathrm{r}}$

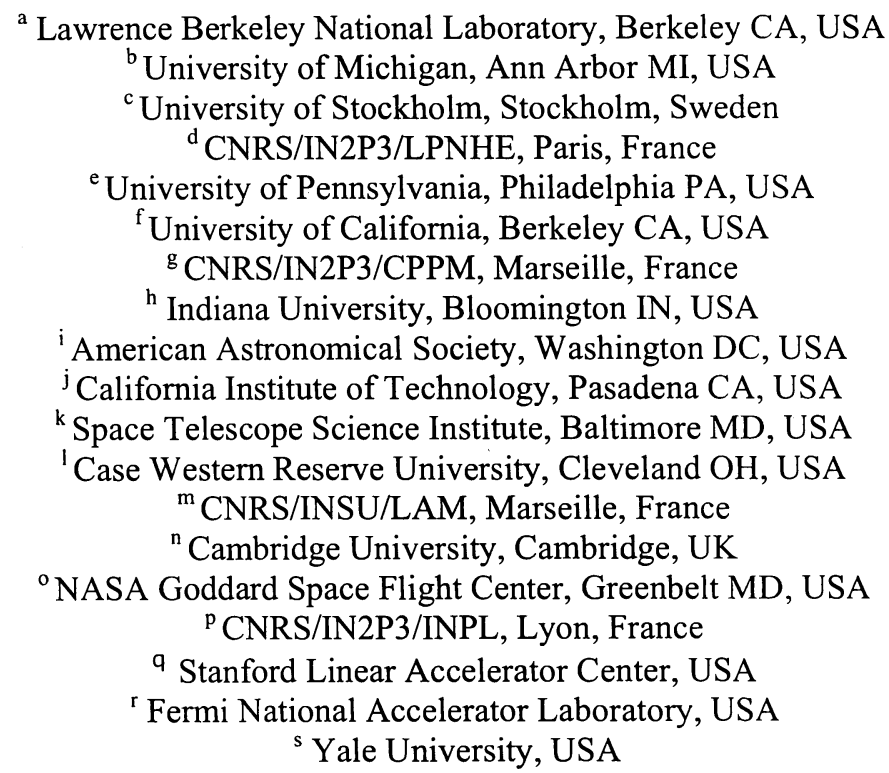

\begin{abstract}
A well-adapted spectrograph concept has been developed for the SNAP (SuperNova/Acceleration Probe) experiment. The goal is to ensure proper identification of Type Ia supernovae and to standardize the magnitude of each candidate by determining explosion parameters. The spectrograph is also a key element for the calibration of the science mission. An instrument based on an integral field method with the powerful concept of imager slicing has been designed and is presented in this paper. The spectrograph concept is optimized to have high efficiency and low spectral resolution ( $\mathrm{R} \sim 100)$, constant through the wavelength range $(0.35-1.7 \mu \mathrm{m})$, adapted to the scientific goals of the mission.
\end{abstract}

Keywords: SNAP, Supernovae, Integral field, Spectrograph, Image slicer 


\section{INTRODUCTION}

The SNAP satellite is designed to measure very precisely the cosmological parameters and to determine the nature of the dark energy. The mission is based on the measurement of some 2000 supernovae ( $\mathrm{SNe}$ ) of Type Ia up to a redshift of $z=1.7$. Details of the mission and the expected physics results can be founded in ${ }^{1,4,5,6}$. Spectroscopy of each candidate supernova near maximum light is required to (a) insure that only Type Ia supernovae are used, and (b) measure the physical parameters of the supernova explosion through various spectral features. The parameters of the explosion, e.g. the progenitor metallicity, have small but important effects upon the peak magnitude and color of Type Ia events, and hence must be measured to insure the highest possible accuracy in determination of the cosmological parameters.

\section{SCIENCE DRIVERS}

To achieve the primary goals of selecting Type Ia supernovae and of controlling for intrinsic physical variations, a spectrum of each candidate is must be acquired near maximum light. Operating in space has clear advantages for such a mission:

× Easy access to the infrared region

$\times \quad$ A stable and small PSF

× 24h operation

$\times$ Lower exposure time, thanks to reduction of background to the level of the zodiacal light

In this case the major limitation is the aperture of the primary mirror $(2 \mathrm{~m})$ and the need for the best overall efficiency to access the faintest supernovae (magnitude $\sim 25$ at peak).

A typical supernova spectrum of Type Ia at peak is shown in Figure 1. Of particular interest is the breadth of all lines, which indicates that high-resolution spectroscopy is not required. The specific signature of Type Ia supernovae is the Sill line at $\lambda=6150 \AA$ (rest frame). This line is very broad ( $\sim 200 \AA$ rest frame) and is broadened further by the redshift factor $(1+z)$ and

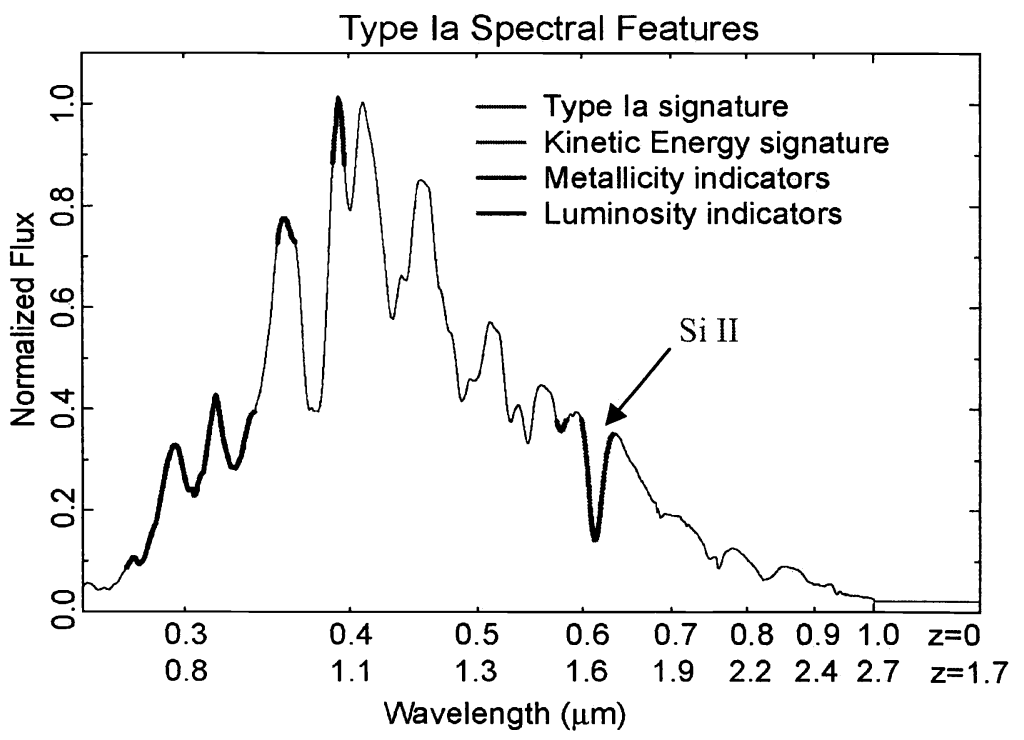

Figure 1: Typical spectrum of SN la.

shifted to $\sim 1.7 \mu \mathrm{m}$ for $z=1.7$. Other types of $\mathrm{SNe}$, such as $\mathrm{II}$ or $\mathrm{Ib}$, have lines of $\mathrm{H}$ or $\mathrm{He}$ in the same wavelength range, allowing the classification of all possible candidates. The feature characteristics (position, width, height, etc.) are directly related to the peak magnitude through physical parameters such as temperature, velocity and progenitor metallicity. In models, the strongest sensitivity to the metallicity in the progenitor system lies in the rest-frame UV band, which defines a broad wavelength range, $0.4<\lambda<1.7 \mu \mathrm{m}$, that must be covered by the instrument. The broad features of the SNe spectra and the non-negligible detector noise contribution for the faintest objects make a lowresolution spectrograph optimal: a resolving power $\lambda / \delta \lambda \sim 100$ at FWHM and 1 pixel per FWHM sampling, with constant resolving power in the $0.6-1.7 \mu \mathrm{m}$ range, is required to keep the $\mathrm{S} / \mathrm{N}$ optimized for all redshifts. The main specifications are summarized in Table 1. 


\begin{tabular}{|l|c|c|}
\hline Property & Visible & IR \\
\hline Wavelength coverage $(\mu \mathrm{m})$ & $0.35-0.98$ & $0.98-1.70$ \\
\hline Field of view & $3.0^{\prime \prime} \times 3.0^{\prime \prime}$ & $3.0^{\prime \prime} \times 3.0^{\prime \prime}$ \\
\hline Spatial resolution element $($ arc sec) & 0.15 & 0.15 \\
\hline Spectral resolution, $\lambda / \delta \lambda$ & 100 & 100 \\
\hline Cumulative throughput & $52 \%$ & $45 \%$ \\
\hline
\end{tabular}

Table 1: Spectrograph main specifications.

The field of view should include the underlying galaxy in order to determine its spectrum during the same exposure. This is necessary for subtraction of the host spectrum from the spectrum in the supernovae region and for an accurate determination of the supernova redshift. A field of view of 3"x 3" covers the mean size of galaxies of redshift 1-2. No strong requirement is specified for pointing. The spectrograph is also a key component of the calibration procedure. The precision required to calibrate the experiment is at the level of $2 \%$, driven by the precision required on the cosmological parameters. The spectrograph will be used to transfer the calibration of fundamental standard stars to primary standards in the range $\mathrm{m}_{\mathrm{V}}=12-18 \mathrm{mag}$ where the imager cannot reach the needed sensitivity. This requires a spectro-photometric calibration at $1 \%$ accuracy.

Space instruments must be light and compact, and must minimize interfaces with the spacecraft. To reduce the time budget, we must take the galaxy and SN spectra in the same exposure and push to the highest efficiency possible for the highest redshifts.

\section{INSTRUMENT CONCEPT TRADE- OFF}

Given the science drivers and requirements, we conducted a trade-off study to choose the best instrument concept. The requirement for simultaneous acquisition of SN and host spectra, and the high object acquisition precision that would be needed for a traditional long slit spectrograph, lead us to prefer a 3D spectrograph. A 3D spectrograph reconstructs the data cube including the two spatial directions $X$ and $\mathrm{Y}$ plus the wavelength direction as shown in Figure 2. For each spatial pixel, the spectrum is reconstructed. Thanks to the 3 " $\times 3$ " field of view, the pointing requirements are relaxed and the galaxy and $\mathrm{SN}$ data are acquired at the same time. Two principal techniques are indicated for 3D spectroscopy: Fourier Transform Spectrometer (FTS), and Integral Field Spectroscopy (IFS).

The FTS technique is based on the classical Michelson interferometer principle. When one of the two flat mirrors is moved, the Fourier transform space of the wavelength is scanned. Herschel is using this technique. But the domain of excellence for the FTS is at longer wavelengths, smaller wavelength range, and higher spectral resolution than SNAP needs. The main drawback to an FTS on SNAP would be the need for a translating device with a quarter-millimeter throw and a positioning accuracy of a few nanometers. This would call for a complex

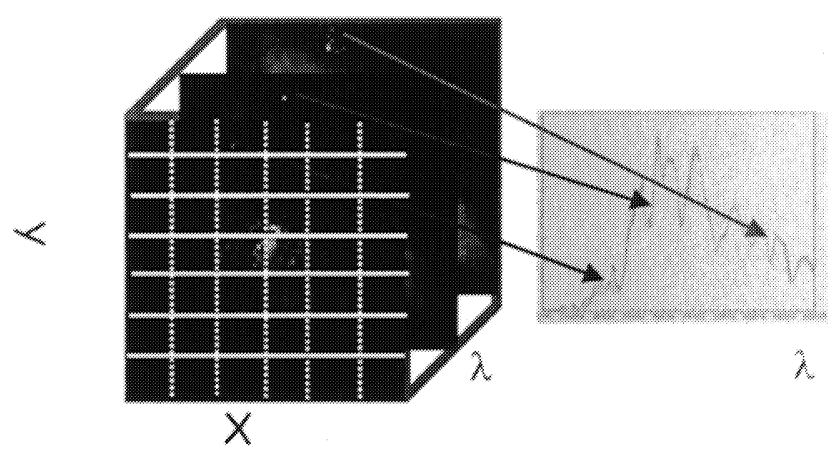

Figure 2: 3D spectroscopy illustration.

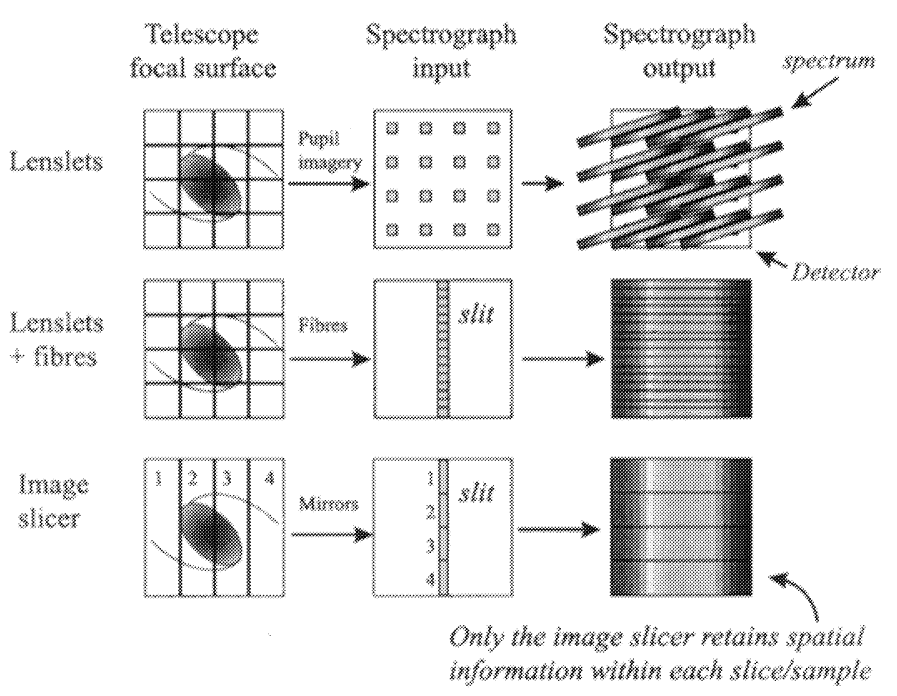

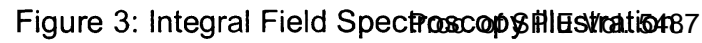


mechanism, even more so for a very precise metrology system.

Integral field spectroscopy using traditional dispersers is based on three generic techniques as shown in Figure 3 . The simplest one is the use of classical microlenses in order to create an image of the telescope pupil at the entrance of the spectrograph $\left(\right.$ Tigre-CFHT ${ }^{9}$ ). The dispersion takes place between the pupil images. As there is little room for each spectrum on the focal plane, a filter wheel is needed. This is excluded for SNAP, where all information should be acquired in one shot. One also has a doubt on the ability to recover diffraction effects which will be dominant in the IR part of the spectrum.

In order to have room to accommodate the spectra, we must rebuild an entrance slit for the spectrograph. This would be possible using microlenses and fibers to convey the light (i.e. VIMOS ${ }^{8,10}$ and GMOS). However, as the coupling efficiency between fibers and microlenses is not optimal, this solution has only an average efficiency. As SNAP is only a 2-meterclass telescope, we must treasure every photon.

The final possibility in our trade-off is the image slicer technique. This technique, developed since 1938 in order to minimize slit losses, is very

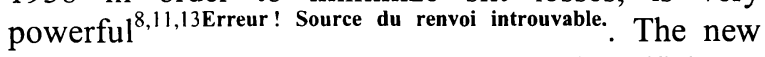
generation of image slicers improves the efficiency and the compactness of the system. Figure 4 shows the principle of this technique. The field of view is sliced along $N$ (in the drawing $N=3$, for SNAP $N=20$ ) strips on a "slicing mirror" (stack of $N$ plates where the active surface is on an edge called a slice). Each of $N$ slices re-images the telescope pupil, creating $N$ telescope pupil images in the pupil plane. Thanks to a tilt adapted to each individual slice, the $N$ pupil images lie along a line. In the pupil plane, a line of "pupil" mirrors is arranged. Each pupil mirror is placed on a pupil image and it re-images the field strip. These images are arranged along a line and form a "pseudo-slit." At this stage, therefore, we have an image of each of the $N$ strips of the field of view. The pseudo-slit is placed in the entrance plane of the



Figure 4. Image slicer principle (courtesy J. Allington-Smith, Durham U.) spectrograph, acting as the entrance slit.

A last line of mirrors is placed on the pseudo-slit. This line adapts the output pupil of the slicer into the input pupil of the spectrograph.

\section{INSTRUMENT CONCEPT}

\section{SYSTEM REQUIREMENTS}

The system requirements for each sub element are summarized on Table 2. To avoid single-point failure in the spectrograph, detectors will be duplicated in the visible and in the NIR focal plane respectively. The field of view is enlarged to $3 " \times 6 "$ and is displayed on 40 slices along the larger dimension. 


\begin{tabular}{|c|c|c|c|}
\hline & Visible & $\overline{\text { NIR }}$ & System description \\
\hline Spatial resolution (arcsec)2 & \multicolumn{2}{|c|}{0.15} & $\begin{array}{l}2 \times 20 \text { Slices } \\
0.9 \times 18 \mathrm{~mm}\end{array}$ \\
\hline $\begin{array}{r}\text { Field-of-View } \\
\text { (arc second) }\end{array}$ & \multicolumn{2}{|c|}{$3 \times 3$} & $3 \times 6$ \\
\hline Wavelength $(\mu \mathrm{m})$ & $0.35-1.0$ & $1.0-1.7$ & Dichroic \\
\hline Spectral Resolution & $100-200$ & $70-100$ & Prism \\
\hline Detector Size & $1 \mathrm{kx} 1 \mathrm{k}$ & $1 \mathrm{kx} 1 \mathrm{k}$ & $\begin{array}{l}800 \times 200 \text { useful } \\
2 \text { booted detectors }\end{array}$ \\
\hline Pixel size & $10-20 \mu \mathrm{m}$ & $18 \mu \mathrm{m}$ & Camera $F / D=12$ \\
\hline Detector Temp $(\mathrm{K})$ & 140 & 140 & Passive cooling \\
\hline
\end{tabular}

Table 2: system specifications

\section{OPTICAL DESIGN}

The optics has been designed to minimize the number of mirrors. This will reduce the risk but will slightly degrade the image quality. A solution with 7 mirrors is currently presented which fulfils a first volume allocation.

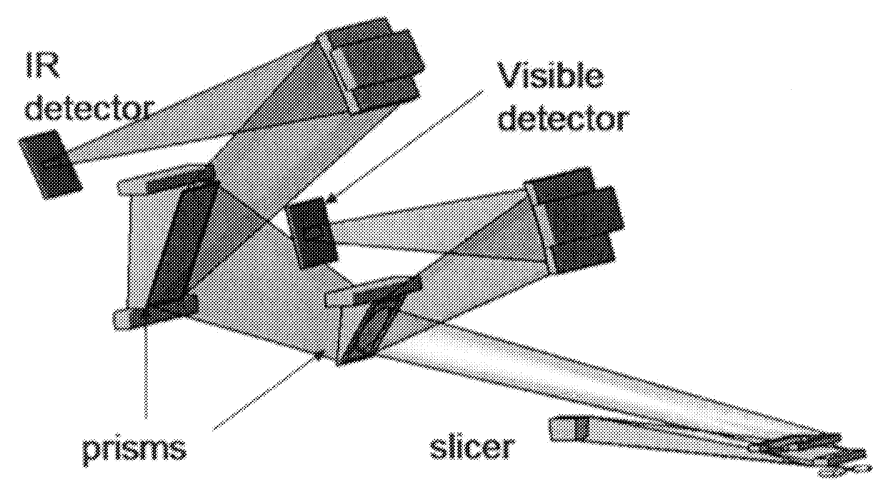

Figure 5: schematic optical design of the spectrograph

A schematic spectrograph optical design is shown on Figure 5 . The beam is going out from the slicer (on the bottom right) to a prism disperser back faced coated by a dichroic. The visible light (blue path) is reflected and the IR beam (in red) continues to a second prism used to reach the required spectral dispersion. The two beams are therefore focused on two detectors. The dimension of the spectrograph is approximately $400 \times 80 \times 100 \mathrm{~mm}$. 


\section{RELAY OPTICS}

This unit is the interface between the telescope beam and the instrument. The optical solution is highly dependent on the implementation of the instrument. The definition of this optical system requires knowledge of the spectrograph position with respect to the telescope focal plane. The beam can be picked off wherever it is most convenient for the overall instrument. It will be beneficial to correct some telescope aberrations within this optical system. A simple, easily conceived three-mirror configuration should be sufficient to satisfy these requirements.

\section{SLICER UNIT}

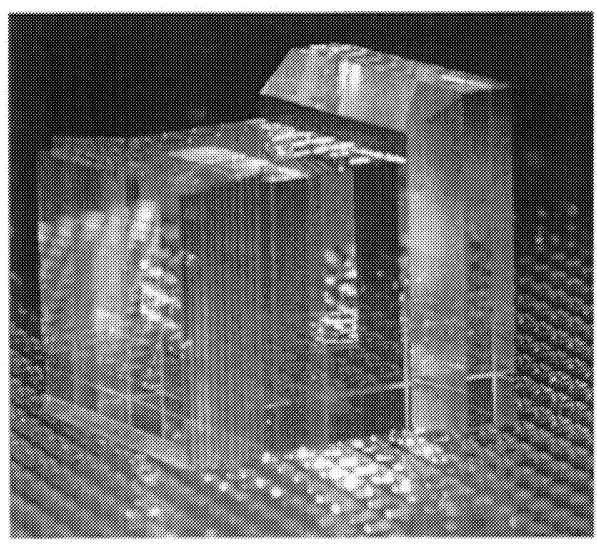

The slicer unit acts as a field reformatting system. As described above, the principle is to slice a 2D field of view into long strips and optically align all the strips to form a long spectrograph entrance slit. The slicing mirror is comprised of a stack of slicers. Each slicer has an optically active spherical surface on one edge (see Figure 6). A line of pupil mirrors does the reformatting. Each pupil mirror sends the beam to a slit mirror, which adapts the pupil to the entrance of the spectrograph. The long thin active surface of each individual slicer will produce a large diffraction effect. In order to minimize flux losses to a few percent, the spectrograph entrance pupil must be oversized. A combined theoretical and experimental approach is underway at LAM to define the optimum entrance pupil (in the infrared bands $1-5 \mu \mathrm{m}$ ).

The baseline requirements on the slicer unit are an accuracy of $\leq \lambda / 10 \mathrm{rms}$ on the optical surfaces and a surface roughness of $\leq 5 \mathrm{~nm}$ rms. Existing prototypes fully meet these specifications.

Figure 6: image of a block of slicers

\section{OPTICAL BENCH}

Thanks to the moderate beam aperture and field of view, the spectrograph optics will be straightforward. The baseline is a classical dichroic spectrograph: The optical beam reaches a BK7 prism with its back face coated with a dichroic. Visible radiation will be reflected while infrared radiation will continue through a second prism, in CaF2. The BK7 prism is used in double pass in the optical domain to reach the required spectral dispersion with a smaller prism. The NIR beam is dispersed by the CAF2 prism in double pass at the requested $\mathrm{R} \sim 100$. Then each beam enters in the appropriated camera which images the spectrum on the visible detector in one side (e.g. $0.35-0.98 \mu \mathrm{m})$ and on the NIR detector in the other side $(0.98-1.70 \mu \mathrm{m})$. Figure 7 shows the actual optical layout.

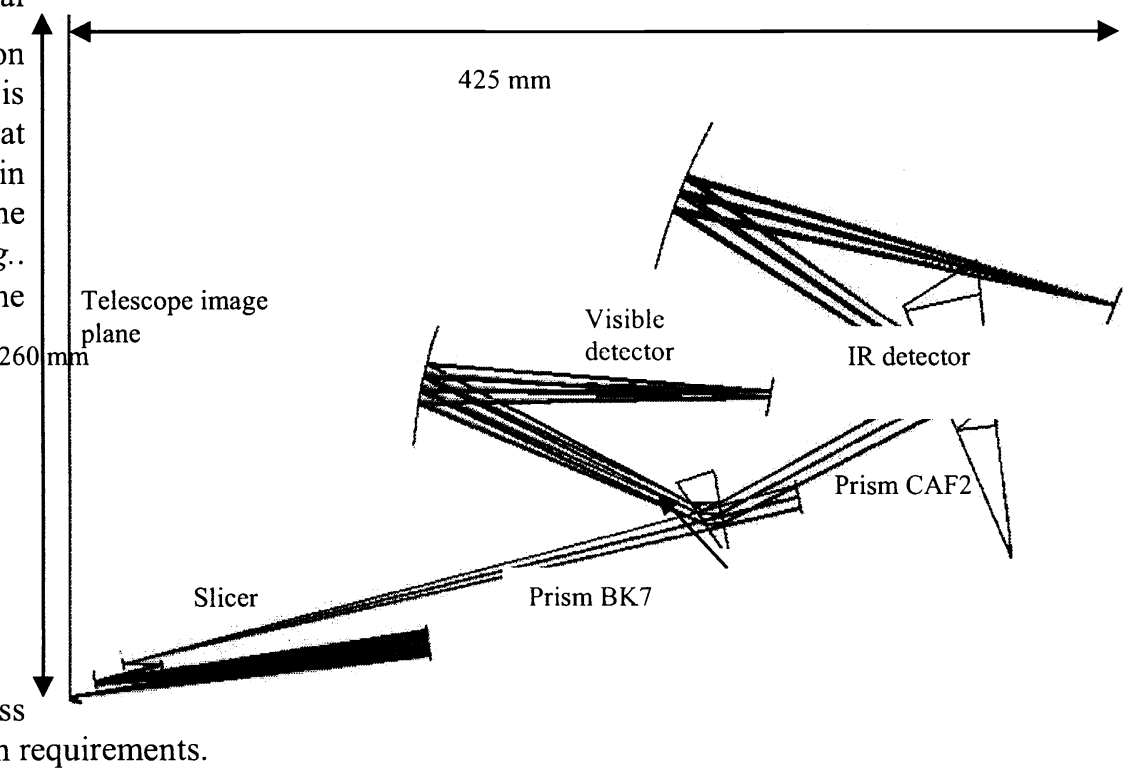

The current design is in agreement with the requirement on image quality and is diffraction limited@1.7 $\mu \mathrm{m}$. Thanks to simulation work, new requirements will be released and first guess is that we are currently over the next iteration in requirements. 


\section{DETECTORS}

In the visible domain, the main goals are high quantum efficiency and very low noises. Given concerns over degradation due to radiation exposure and the poor performance of conventional thinned CCDs in the red part of the visible, we will study carefully the applicability of the LBNL CCDs ${ }^{7}$. Thinned, backsideilluminated, low-noise conventional CCDs of $1024 \times 1024$ pixels are an alternative option.

In the IR, some factors constrain the detector technologies. The overall temperature for the SNAP

\begin{tabular}{|l|c|c|}
\hline & Visible & IR \\
\hline Detector size & $1 \mathrm{k} \times 1 \mathrm{k}$ & $1 \mathrm{k} \times 1 \mathrm{k}$ \\
\hline Pixel size & $15-20 \mu \mathrm{m}$ & $18 \mu \mathrm{m}$ \\
\hline Detector temperature(K) & 140 & 140 \\
\hline$<\mathrm{QE}>(\%)$ & 80 & 60 \\
\hline Read noise(e) & 2 & 5 \\
\hline Dark current(e/pixel/s) & 0.001 & 0.02 \\
\hline
\end{tabular}

Table 3: detector specifications instruments will be fixed in the range 130-140 K and the spectrograph must operate in this range. While keeping noise figures low, the cutoff wavelength of the array must be as close as possible to $1.7 \mu \mathrm{m}$. A $1024 \times 1024 \mathrm{HgCdTe}^{6}$ array with $18 \mu \mathrm{m}$ pixels from Rockwell is under consideration. The choice of visible and IR detectors will be done in close collaboration with the teams designing the SNAP imager in order to maintain the simplest overall solution for SNAP. A detailed list of the performance specifications for the detectors is provided in Table 3. To achieve the listed performance in read noise and dark current, a multiple sampling technique is required. The impact of the rate of cosmic rays on the readout noise is under study.

\section{SIMULATION AND PERFORMANCES}

\section{SIMULATION}



Figure 8: image of a SN+ underlayed galaxy on the detector

The simulation is then used to estimate the expected performances of the instrument.
An optical system has been developed, based on Fourier optics. It is coupled to the detailed design of the instrument via Zernike coefficients produced by the Zemax program. The output is a discrete psf at the detector level, for a monochromatic point source at a given position. To generate data for a full detector, a parameterisation of the psf has been implemented with shapelet coefficients which depend of the spatial position and of the wavelength. This enables us to simulate realistic data cubes for a $\mathrm{SN}$ and its companion () and of SN spectrum in the $0.35-1.7$ um range (Figure 8)

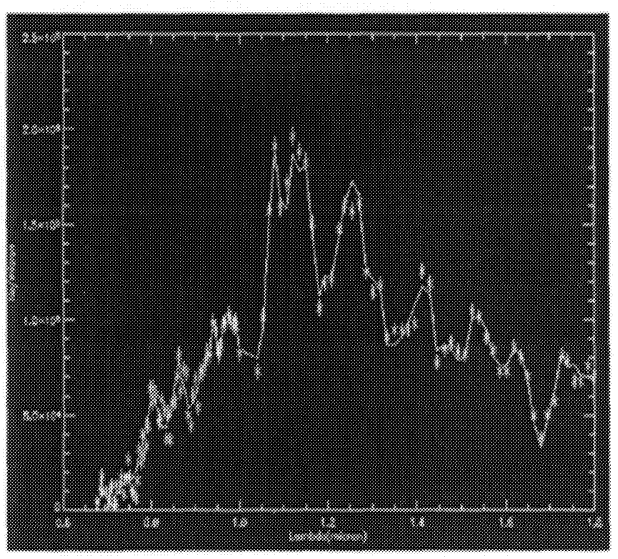

Figure 9: $S N$ spectrum at $z=1.7$ 


\section{SPECTRAL RESOLUTION}

The spectral resolution is shown on Figure 10 and has been optimised to be maintained as flat as possible, privileging lower values in the IR region. The detailed simulation has been used to confirm this resolution.
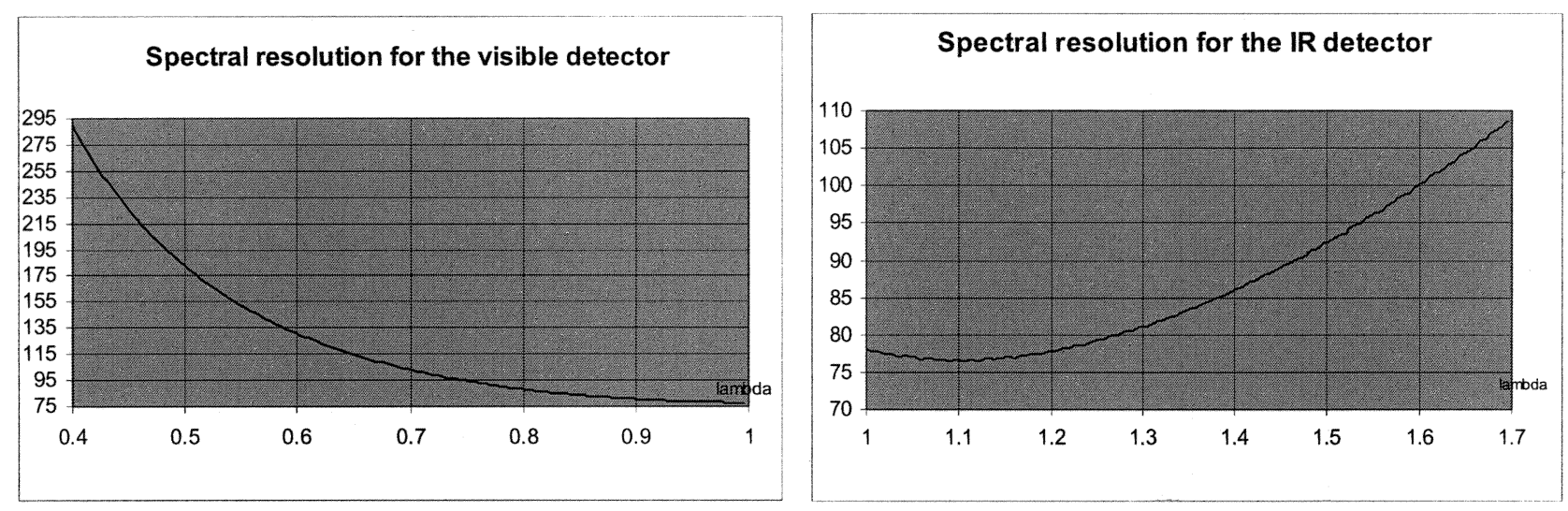

Figure 10: spectral resolution in the visible and IR

\section{THROUGHPUT}

The estimated throughput of the instrument is given on Table 4. Thanks to the good throughput of the reflective silver coated optic and to the slicer performance, the expected total throughput of the instrument is 3 to 4 time higher than the HST-STIS one. This table was derived from the full simulation of the slicer and the spectrograph including the diffraction and aberration effects on each optical plane.

\begin{tabular}{|l|l|l|l|}
\hline & \# elements & $\begin{array}{l}\text { Efficiency } \\
\text { /elements }\end{array}$ & $\begin{array}{l}\text { Cumulative } \\
\text { efficiency }\end{array}$ \\
\hline Telescope & 4 & 0,98 & 0.92 \\
\hline Relay optic & 3 & 0,98 & 0.87 \\
\hline $\begin{array}{l}\text { Slicer } \\
\text { (mirrors+straylight+diffraction) }\end{array}$ & 3 & 0.88 & 0.77 \\
\hline Spectro & Mirrors 1 & 0.98 & \\
& Prism & 0.88 & \\
& Dichroic & 0.88 & 0.58 \\
\hline Detector vis & 1 & 0.9 & 0.52 \\
\hline Detector IR & 1 & 0.8 & 0.46 \\
\hline
\end{tabular}

Table 4: instrument efficiency estimation 


\section{PRINCIPAL R\&D EFFORT: THE SLICER UNIT}

The proposed image slicer is of the same type as the one studied in the context of the NGST near-IR spectrograph ${ }^{14}$ (Allington-Smith et al., 1999; Le Fèvre et al., 1999). This technology has been ranked at NASA readiness level (TRL) 5 by a panel of NASA experts in the context of the concept appraisal of pre-phase A NGST studies. Readiness level 6 is required to be "space qualified." Prototyping activities at Laboratoire d'Astrophysique de Marseille (LAM), in collaboration with other European institutes are under way to validate this technology both for large ground-based telescopes and for space applications, under funding by various agencies including ESA, CNRS and CNES. ESA is funding a consortium lead by LAM and including Observatoire de Lyon, Durham University, and ESO, in order to push the slicer technology up to TRL 6. The development is ongoing for JWST with a slicer prototype and will fully validate this technique for the SNAP application. The prototype represents a realistic unit for a space-qualified application based on Zerodur-glass slices. Several slices have been manufactured successfully to specifications and will be qualified for space instrumentation within this year ${ }^{15}$.

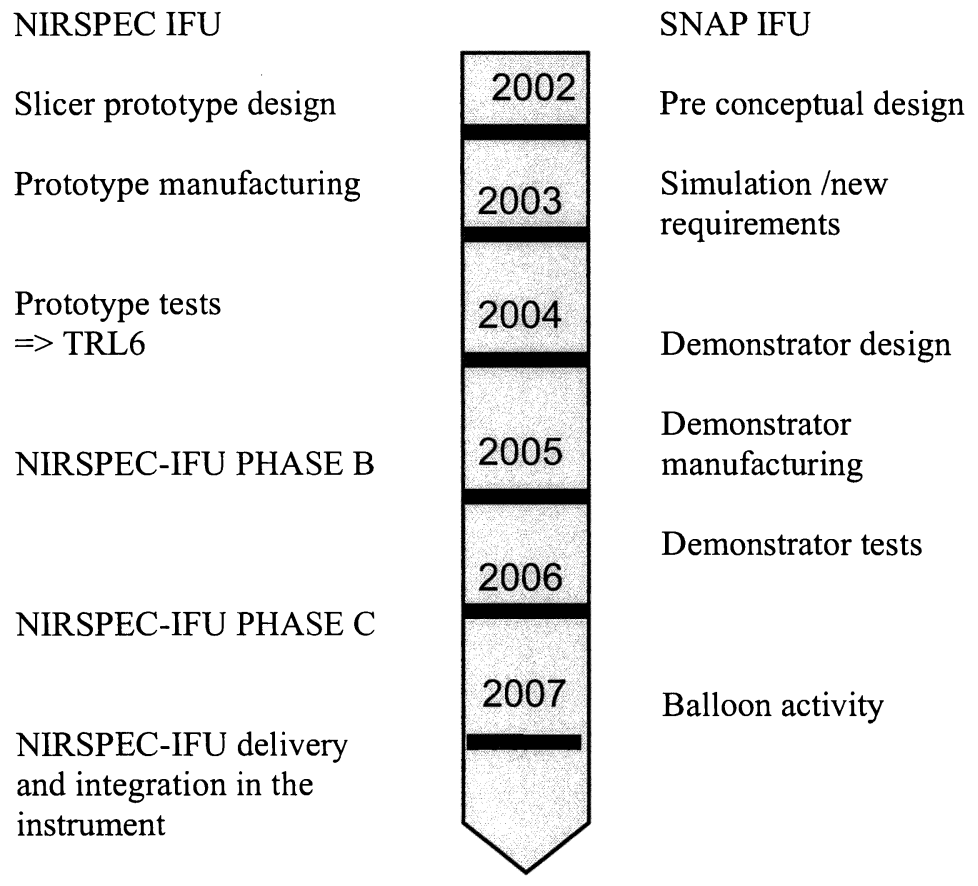

Figure 11: Development Road map

The R\&D effort necessary to adapt this concept to the SNAP requirements therefore meshes nicely with ongoing activities and will be synchronized with the R\&D phase. The present prototype can adequately represent the SNAP application. The road map of the R\&D is presented in Figure 11. To validate furthermore the concept developed for SNAP, an optical bench using the slicer prototype will be funded to evaluate the optical performances and to test the ability of an accurate spectro-photometric calibration. A program to evaluate the calibration procedure for the SNAP mission in under work and will possibly include a balloon to measure IR targets ${ }^{16}$. We will propose to study the possibility to include an integral field spectrograph with a slicer and adapted specifications ( as $\mathrm{R}=3000$ to solve $\mathrm{OH}$ emission etc...). 
Other contributions to this meeting provide details of different image slicer developments, design, performance studies, and other projects related to slicer technology.

\section{CONCLUSION}

Spectral observations and accurate calibration are one of the key programs of the SNAP mission. For a reasonable budget, volume, and risk, the proposed concept will achieve the objectives of the mission within the time allocation proposed in the mission strategy. Thanks to the European effort in the domain of image slicers, the project will benefit from the most efficient technique for 3D spectroscopy. The maturity of this technique is increasing and will be at Technical Readiness Level 6 (NASA scale) by mid 2004. The SNAP project will benefit fully from this advance. The R\&D effort for the spectrograph will be focused on the image slicer we are already building for ESA for JWSTNIRSPEC and with a small adaptation, will be used to validate the concept developed for the SNAP mission. 


\section{REFERENCES}

1. G. Aldering et al, SNAP Collaboration, 2002, SPIE Proc , $\mathbf{4 8 3 5 , 2 1 .}$

2. A. G. .Kim et al, SNAP Collaboration, 2002, SPIE Proc.,4836.

3. Bebek,C.J. et al, SNAP Collaboration, 2003, SPIE Proc., 5164,74

4. M.L. Lampton, SNAP Collaboration, 2002b, SPIE Proc., 4849,215.

5. M.L. Lampton, SNAP Collaboration 2003, SPIE Proc. , 5166.

6. G. Tarlé et al. SNAP NIR , 2002, SPIE Proc., 4850,919.

7. Bebek et al, 2002, SPIE Proc., 4669,161

8. C. Bonneville, La Spectroscopie Intégrale de Champ : étude et réalisations, chapitre I, Thèse de doctorat, Université d'Aix-Marseille III, 2002.

9. R. Bacon, G. Adam, A. Baranne, G. Courtes, D. Dubet, J.P. Dubois, E. Emsellem, P. Ferruit, Y. Georgelin, G. Monnet, E. Pecontal, A. Rousset, F. Sayede, "3D spectrography at high spatial resolution. Concept and realisation of the integral field spectrograph TIGER", A\&Asuppl, 113, pp. 347, 1995.

10. C. Bonneville, E. Prieto, O. Le Févre, M. Saisse, C. Voet, A. Zanichelli, B. Garilli, G. Vettolani, J.-P. Picat, D. Mancini, D. Maccagni, "VIRMOS very wide integral field unit for the VLT: integration and performances", SPIE proc., 4841, 2002.

11. I.S. Bowen, "The image slicer, a device for reducing loss of light at slit of stellar spectrograph", Ap. J., 88, pp. 113, 1938.

12. Bonneville, C.; Prieto, E.; Design, prototypes, and performances of an image slicer system for integral field spectroscopy; Specialized Optical Developments in Astronomy. Edited by Atad-Ettedgui, Eli; D'Odorico, Sandro. Proceedings of the SPIE, Volume 4842, pp. 162-173 (2003)

13. R. Content, “A New Design for Integral Field Spectroscopy with 8-m Telescopes”, SPIE Proc., 2871, pp. 1295, 1997.

14. Prieto, E \& al; Great opportunity for NGST-NIRSPEC: a high-resolution integral field unit; IR Space Telescopes and Instruments. Edited by John C. Mather . Proceedings of the SPIE, Volume 4850, pp. 486-492 (2003)

15. Prieto, E \& al; Slicer manufacturing and applications. Brazilian Instrumentation Workshop, Brazil, Angra dos Reïs, 2003, in press

16. S.Deustua et al., SNAP Collaboration, 2003, SPIE Proc, 5164,84. 